\title{
INTEGRATING PRE AND POST-EMERGENCE HERBICIDES FOR CONTROLLING WEEDS IN DRILL-SEEDED RICE (ORYZA SATIVA L.)
}

\author{
ABD EL-NABY, S. S. M.; I. H. ABOU EL-DARAG and \\ A. M. A. EL-GHANDOR
}

Rice Dept., Field Crops Research Institute, ARC, Giza, Egypt.

(Manuscript received 6 November 2017)

\begin{abstract}
$\mathrm{T}$ wo field experiments were carried out at the experimental farm of Sakha Agricultural Research Station, Kafrelsheikh, Egypt during the summer seasons of 2015 and 2016 to find out the optimum pre and post-emergence herbicide treatments from the recommended herbicides to control weeds under drillseeded rice conditions (Giza179 cv). Fourteen weed control treatments included two pre-emergence herbicides i.e. thiobencarb $50 \%$ at the rate of $3.57 \mathrm{~kg}$ ai ha- ${ }^{-1}$ alone and followed by six weed control treatments included; penoxsulam $2.5 \%$ at $0.0238 \mathrm{~kg}$ ai ha${ }^{1}$, bispyribac-sodium $2 \%$ at $0.0381 \mathrm{~kg}^{2}$ ai ha- ${ }^{-1}$ and fenoxaprop-ethyl $7.5 \%$ at $0.0625 \mathrm{~kg}^{2}$ ai ha-1 alone and mixed with halosulfuron $75 \%$ at $0.0357 \mathrm{~kg}$ ai ha-1, also pendimethalin $50 \%$ at rate of $2.023 \mathrm{~kg}$ ai $\mathrm{ha}^{-1}$ alone and followed by the previous six post-emergence weed control treatments as compared to weedy check (untreated). Randomized Complete Block Design (RCBD) with three replications was used. The results revealed that, spraying pendimethalin as pre-emergence herbicides at 4 days after seeding (DAS) recorded the best weed control, highest rice growth characters and grain yields, as compared to thiobencarb. Each of penoxsulam $2.5 \%$ or bispyribac-sodium $2 \%$ or fenoxaprop-ethyl $7.5 \%$ mixed with halosulfuron $75 \%$ gave the lowest dry weights of grasses, broadleaves and total weeds in addition to rice dry biomass, grain yield and its studied attributes. Based on obtained results, it can be concluded that pendimethalin as pre-emergence herbicide at 4 DAS followed by spraying any of penoxsulam $2.5 \%$ or bispyribac-sodium $2 \%$ or fenoxaprop-ethyl $7.5 \%$ mixed with halosulfuron $75 \%$ at recommended doses applied at 15, 22 and 35 DAS, respectively were the best combinations for controlling weeds and gave the highest grain yield in drill-seeded rice.
\end{abstract}

Keywords: Rice, drill-seeded, grasses, broadleaves, weeds, herbicide, pre-emergence, post- emergence and weed control.

\section{INTRODUCTION}

Rice (Oryza sativa L.) is one of the main cereal crops in Egypt, in addition, its importance as a main dish for most of Egyptians. It is a stable food for more than half of the world population (FAOSTAT, 2011). In Egypt, rice productivity has been increased from 5.712 tons ha-1 in 1984 to 9.50 tons ha-1 $^{-1}$ in 2014 (FAOSTAT, 2014) by releasing high yielding capacity varieties, efficient weed control, optimum cultural practices and integrated pest (diseases and insects) management (IPM). 
Direct seeded rice (DSR) cultivation save water, seeds and low production cost as compared with traditional transplanting (Hongyan et al., 2014). Because of water shortage and unwilling rural work in Egypt, scientists must develop strategies to increase sustainability and profitability of direct seeded-rice systems as drill-seeded rice. Moreover effective weed management practices must be established to maximize rice yield per unit area.

Weed control is the most serious problem and challenge force both of drill and broadcast-seeded rice, weeds can directly or indirectly negatively affect rice yield (Mahajan et al., 2009). Weed flora in drill-seeded rice is a complex of mainly grassy weeds (Echinochloa crus-galli, E. colona and Dinebra retroflexa) and broad leave weeds so that, individual herbicide application through rice growth season in drilling cultivation is not effective. This is because of the ability of some weeds to still life and/or had a quick recovery again after herbicide application in addition to, the new generations of weeds which can be grow during different times in the same season. Moreover, herbicide tolerance can occur because of frequent application for the same herbicide at the same area every year.

In drill-seeded rice, weeds can cause a huge reduction in yield of rice determined by $14-93 \%$ as compared to transplanted rice (17-47\%) (Ranjit, 1997). In addition, decreasing grain quality and increasing rice production costs. El-Refaee et al., 2006 reported that drill-seeded rice method had four weeks of flush irrigation during the crop establishment period which resulted in large accompanied weed flora. There are few limiting factors associated with DSR that impair yields including cropweed competition. Therefore, there is a scope to increase yield by adopting integrated weed management approaches including tillage systems, competitive cultivars, use of crop residue as mulch, hand hoeing, and herbicides in DSR (Chauhan, 2012).

Chemical weed control is the key of high yielding under direct-seeded rice (DSR) system, it must be a sequential application of pre and post-emergence herbicides to achieve the best weed control and rice yield as superior grain quality (Chauhan et al., 2015) besides avoiding the appearance of tolerant-herbicide weeds rapidly. Singh et al., 2016 found that the application of pendimethalin $f b$ bispyribacsodium + azimsulfuron achieved the maximum reduction in weed biomass and recorded the best weed control efficiency (WCE) percentages. These results are similar to those obtained by Ganie et al., 2014 and Awan et al., 2015.

The objective of the present study is to investigate the integration between both pre and post-emergence herbicides for wide spectrum of weed management in drill-seeded rice to achieve the best weed control, this will reflect on a good rice growth and better yield. 


\section{MATERIALS AND METHODS}

Two field experiments were carried out during 2015 and 2016 seasons at Sakha Agricultural Research Station, ARC, Egypt. Giza 179 as a new released rice cultivar was planted using drilling machine at seed rate of $120 \mathrm{~kg} \cdot \mathrm{ha}^{-1}$ at $20^{\text {th }}$ of May in both seasons of study (the optimum date of sowing as recommended). Plot size was $14 \mathrm{~m}^{2}(4 \mathrm{~m} \times 3.5 \mathrm{~m})$. Randomized Complete Block Design (RCBD) with three replications was used. The normal rice agricultural practices were applied as recommended for drill-seeded rice. Weed control treatments were suggested as follow:

1- Pendimethalin 50\% EC (Stomp) at $2.023 \mathrm{~kg}$ ai ha-1.

2- Thiobencarb 50\% EC (Saturn) at $3.57 \mathrm{~kg}$ ai ha-1.

3- Pendimethalin $50 \%$ EC followed by penoxsulam $2.5 \%$ OD (Rainbow) at rate of $0.0238 \mathrm{~kg}$ ai ha-1.

4- Pendimethalin $50 \%$ EC followed by bispyribac-sodium 2\% SL (Nominee 2\%) at rate of $0.0381 \mathrm{~kg}$ ai ha-1.

5- Pendimethalin 50\% EC followed by fenoxaprop-ethyl 7.5\% EW (Whipsuper) at rate of $0.0625 \mathrm{~kg}$ ai ha-1.

6- Thiobencarb $50 \%$ EC followed by penoxsulam $2.5 \%$ OD (Rainbow) at rate of $0.0238 \mathrm{~kg}$ ai ha-1.

7- Thiobencarb $50 \%$ EC followed by bispyribac-sodium 2\% SL (Nominee $2 \%$ ) at rate of $0.0381 \mathrm{~kg}^{\mathrm{ai} \mathrm{ha}}{ }^{-1}$.

8- Thiobencarb $50 \%$ EC followed by fenoxaprop-ethyl 7.5\% EW (Whipsuper) at rate of $0.0625 \mathrm{~kg}$ ai ha-1.

9- Pendimethalin $50 \%$ EC followed by penoxsulam+ halosulfuron-methyl $75 \%$ WG (Inpul) at rate of $(0.0238+0.0357 \mathrm{~kg}$ ai ha-1 $)$.

10- Pendimethalin $50 \%$ EC followed by bispyribac + halosulfuron-methyl $75 \%$ WG at rate of $(0.0381+0.0357 \mathrm{~kg}$ ai ha-1 $)$.

11- Pendimethalin 50\% EC followed by fenoxaprop + halosulfuron-methyl 75\%WG at rate of $\left(0.0625+0.0357 \mathrm{~kg}^{2}\right.$ ai ha-1).

12- Thiobencarb $50 \%$ EC followed by penoxsulam+ halosulfuron-methyl $75 \%$ WG (Inpul) at rate of $(0.0238+0.0357 \mathrm{~kg}$ ai ha-1).

13- Thiobencarb 50\% EC followed by bispyribac + halosulfuron-methyl $75 \%$ WG at rate of $(0.0381+0.0357 \mathrm{~kg}$ ai ha-1 $)$.

14- Thiobencarb $50 \%$ EC followed by fenoxaprop + halosulfuron-methyl $75 \%$ WG at rate of $\left(0.0625+0.0357 \mathrm{~kg}\right.$ ai ha-1 $\left.{ }^{-1}\right)$.

15- Weedy check (untreated). 
Thiobencarb and pendimethalin as pre-emergence herbicides were sprayed in 300 liter water per hectare on wet land at 4 days after seeding (DAS) by using Knapsack sprayer then the soil was flush irrigated after 24 hours from herbicidal application.

Penoxsulam alone or mixed with halosulfuron were applied at 15 DAS, while bispyribac-sodium alone or mixed with halosulfuron were sprayed at 22 DAS, then fenoxaprop-ethyl alone or mixed with halosulfuron treatments were applied at 35 DAS. All post-emergence weed control treatments were sprayed in 300 liter water per hectare on wet land by using Knapsack sprayer then the soil was flush irrigated after 24 hours from herbicidal application.

At 80 DAS, weeds were sampled by area of $50 \times 50 \mathrm{~cm}$ quadrate replicated four times for each plot, weeds were cleaned then air dried then oven dried to stable weight, dry weights per square meter for each weed species were recorded then total dry weights of total weeds were calculated.

Also, rice dry weight was measured by the same method. Before harvest, panicles were counted in two random quadrates of $50 \times 50 \mathrm{~cm}$ and number of panicles per square meter was recorded. After rice maturity, the central $5 \mathrm{~m}^{2}$ from each plot were manually harvested to determine grain yield then recorded rice grain yield at $14 \%$ moisture content.

Data analysis: the collected data were subjected to proper statistical analysis of variance according to Snedecor and Cochran (1971). Weed data were statistically analyzed by MSTATC program after transformed according to square-root transformation $(\sqrt{ }[x+0.5])$, while rice collected data were directly analyzed by MSTATC program then the means of both weeds and rice characters were compared by using Duncan's Multiple Range Test (Duncan, 1955).

\section{- Correlation coefficient:}

Phenotypic correlation coefficients between all possible combinations of pair of characters were computed as follows:

$$
\mathbf{r}=\frac{\Sigma \mathrm{XY}-\Sigma \mathrm{X} \cdot \Sigma \mathrm{Y} / n}{\left[\Sigma(\mathrm{X}-\overline{\mathrm{X}})^{2} \cdot \Sigma(\mathrm{Y}-\overline{\mathrm{Y}})^{2}\right]^{1 / 2}}
$$

The significance of the $(r)$ values tested by using the (t) test at 0.05 and 0.01 levels of significance by Steel and Torrie (1960) as follows:

Calculated $(t)$ value for $(r)=r\left(n-2 / 1-r^{2}\right)^{1 / 2}$.

The estimated $(t)$ values for the different correlation coefficients were tested against tabulated $(t)$ values with $(n-2)$ degrees of freedom at 0.05 and 0.01 levels of significance, where $(n)$ is the number of error degrees of freedom. 
Table 1. Studied herbicides trade name, rate per feddan, active ingredient, rate $\mathrm{Kg}$ ai ha-1 ${ }^{-1}$ chemical group, molecular formula, site of action and target weeds.

\begin{tabular}{|c|c|c|c|c|c|c|c|}
\hline $\begin{array}{l}\text { Herbicide } \\
\text { trade name }\end{array}$ & $\begin{array}{l}\text { Rate } \\
\text { fed }^{-1}\end{array}$ & $\begin{array}{c}\text { Active } \\
\text { ingredient } \\
\text { (ai) }\end{array}$ & $\begin{array}{c}\text { Rate } \\
\text { (Kg ai ha- } \\
1 \text { ) }\end{array}$ & Chemical group & $\begin{array}{l}\text { Molecular } \\
\text { formula }\end{array}$ & Site of Action & Target weeds \\
\hline Saturn $50 \%$ EC & 3 Lit. & thiobencarb & 3.57 & Thiocarbamate & $\mathrm{C}_{12} \mathrm{H}_{16} \mathrm{CINOS}$ & $\begin{array}{c}\text { Systemic - } \\
\text { photosynthesis inhibitors }\end{array}$ & Grassy + sedges \\
\hline Rainbow $2.5 \%$ OD & $\begin{array}{l}400 \\
\mathrm{ml} .\end{array}$ & penoxsulam & 0.0238 & Triazolopyrimidine & $\mathrm{C}_{16} \mathrm{H}_{14} \mathrm{~F}_{5} \mathrm{~N}_{5} \mathrm{O}_{5} \mathrm{~S}$ & $\begin{array}{c}\text { Systemic - } \\
\text { ALS inhibitors }\end{array}$ & $\begin{array}{l}\text { Grassy + broad } \\
\text { leaves }+ \text { sedges }\end{array}$ \\
\hline Nominee 2\% SL & $\begin{array}{l}800 \\
\mathrm{ml} .\end{array}$ & $\begin{array}{l}\text { bispyribac- } \\
\text { sodium }\end{array}$ & 0.0381 & $\begin{array}{l}\text { Pyrimidinyloxybenzoic } \\
\text { acid herbicides }\end{array}$ & $\mathrm{C}_{19} \mathrm{H}_{17} \mathrm{~N}_{4} \mathrm{NaO}_{8}$ & $\begin{array}{l}\text { Systemic - } \\
\text { ALS inhibitors }\end{array}$ & Grassy + sedges \\
\hline $\begin{array}{l}\text { Whip-super } 7.5 \% \\
\text { EW }\end{array}$ & $\begin{array}{l}350 \\
\mathrm{ml} .\end{array}$ & $\begin{array}{c}\text { fenoxaprop- } \\
\text { ethyl }\end{array}$ & 0.0625 & $\begin{array}{l}\text { Aryloxyphenoxypropionic } \\
\text { herbicides }\end{array}$ & $\mathrm{C}_{18} \mathrm{H}_{16} \mathrm{ClNO}_{5}$ & $\begin{array}{l}\text { Systemic -inhibition of acetyl } \\
\text { CoA carboxylase (ACCase) }\end{array}$ & Grassy weeds \\
\hline Stomp $50 \%$ EC & $\begin{array}{l}1.7 \\
\text { Lit. }\end{array}$ & pendimethalin & 2.023 & Dinitroaniline & $\mathrm{C}_{13} \mathrm{H}_{19} \mathrm{~N}_{3} \mathrm{O}_{4}$ & $\begin{array}{l}\text { Microtubule assembly } \\
\text { inhibitor }\end{array}$ & $\begin{array}{c}\text { Grassy + broad } \\
\text { leaves }\end{array}$ \\
\hline Inbul 75\% WG & $20 \mathrm{~g}$. & $\begin{array}{l}\text { halosulfuron- } \\
\text { methyl }\end{array}$ & 0.0357 & $\begin{array}{l}\text { pyrimidinylsulfonylurea } \\
\text { herbicides }\end{array}$ & $\mathrm{C}_{12} \mathrm{H}_{13} \mathrm{ClN}_{6} \mathrm{O}_{7} \mathrm{~S}$ & $\begin{array}{l}\text { Systemic - } \\
\text { ALS inhibitors }\end{array}$ & $\begin{array}{l}\text { Broad leaves + } \\
\text { sedges }\end{array}$ \\
\hline
\end{tabular}

Fed. $=$ feddan $\left(4200 \mathrm{~m}^{2}\right)$, Lit. = litter, ha $=$ hectare $\left(10000 \mathrm{~m}^{2}\right), \mathrm{g}=$ gram, ALS $=$ acetolactate synthase 


\section{RESULTS AND DISCUSSION}

\section{A- Weeds:}

The major weed species associated with rice crop during the two growing seasons were grassy weeds including; Echinochloa crus-galli (barnyard grass) and Echinochloa colona (jungle rice) and broad leave weeds including (Ammania baccifera) and others. Dry weights per square meter for each weed species were recorded then total dry weights of total weeds were calculated and used as reliable indicators for weed distribution in rice plots.

\section{A.1. Effect of pre and post-emergence weed control treatments on dry weights of grassy weeds, broadleaves and total weeds during 2015 and 2016 seasons.}

Data on dry weights $\left(\mathrm{g} \cdot \mathrm{m}^{-2}\right.$ ) of grassy weeds, broadleaves and total weeds as affected by pre and post-emergence weed control treatments in 2015 and 2016 seasons are presented in Table (2). Pendimethalin application (2.023 Kg ai ha $\left.{ }^{-1}\right)$ at 4 DAS followed by penoxsulam $(0.0238 \mathrm{Kg}$ ai ha-1) + halosulfuron $(0.0357 \mathrm{Kg}$ ai ha-1) or bispyribac-sodium $(0.0381 \mathrm{Kg}$ ai ha-1 $)+$ halosulfuron $\left(0.0357 \mathrm{Kg}^{-1} \mathrm{ha}^{-1}\right)$ as well as fenoxaprop $\left(0.0625 \mathrm{Kg}_{\text {ai ha}}{ }^{-1}\right)+$ halosulfuron $\left(0.0357 \mathrm{Kg}\right.$ ai ha- $\left.{ }^{-1}\right)$ treatments achieved the best weed control (lowest values of grasses, broadleaves and total weeds dry weights) for studied traits in the first season without significant differences between thiobencarb at rate of $3.57 \mathrm{Kg}_{\text {ai ha-1 }}{ }^{-1}$ at 4 DAS followed by penoxsulam $(0.0238 \mathrm{Kg}$ ai $\left.\mathrm{ha}^{-1}\right)+$ halosulfuron $\left(0.0357 \mathrm{Kg}\right.$ ai ha- $\left.{ }^{-1}\right)$ or bispyribac-sodium $\left(0.0381 \mathrm{Kg}\right.$ ai ha-1 ${ }^{-1}+$ halosulfuron $\left(0.0357 \mathrm{Kg}\right.$ ai ha-1) as well as fenoxaprop $\left(0.0625 \mathrm{Kg}\right.$ ai ha-1 ${ }^{-1}+$ halosulfuron $(0.0357 \mathrm{Kg}$ ai ha-1) through 2016 season. While untreated plots (weedy check) scored the highest values of grasses, broadleaves and total weeds dry weights in the two growing seasons. Previous studies showed that bispyribac-sodium mixed with broadleaves herbicide recorded better weed control than the single application of bispyribac-sodium because of the mixture can be manage both grassy and broadleaves weeds in the same time to reduce weed-competition against rice for water, nutrients and light (Chauhan et al., 2013 and Mahajan and Chauhan, 2015). 
Table 2. Dry weight of grassy weeds, broadleaves and total weeds $\left(\mathrm{g} \mathrm{m}^{-2}\right)$ as affected by weed control treatments during 2015 and 2016 seasons. Weed data were subjected to square-root $(\sqrt{ }[x+0.5])$ transformation before analysis; transformed values are shown in parentheses.

\begin{tabular}{|c|c|c|c|c|c|c|c|c|}
\hline \multirow[b]{2}{*}{ Treatment } & \multirow[b]{2}{*}{$\begin{array}{c}\text { Rate } \\
\left(\mathrm{Kg} \text { ai ha }{ }^{-1}\right)\end{array}$} & \multirow[b]{2}{*}{$\begin{array}{l}\text { Time of } \\
\text { Application } \\
\text { (DAS) }\end{array}$} & \multicolumn{3}{|c|}{2015 season } & \multicolumn{3}{|c|}{2016 season } \\
\hline & & & $\begin{array}{c}\text { Grassy weeds } \\
\text { dry weight } \\
\left(\mathrm{g} \mathrm{m}^{-2}\right)\end{array}$ & $\begin{array}{c}\text { Broadleaves } \\
\text { dry weight } \\
\left(\mathrm{g} \mathrm{m}^{-2}\right)\end{array}$ & $\begin{array}{l}\text { Total weeds } \\
\text { dry weight ( } \mathrm{g} \\
\mathrm{m}^{-2} \text { ) }\end{array}$ & $\begin{array}{c}\text { Grassy weeds } \\
\text { dry weight } \\
\left(\mathrm{g} \mathrm{m}^{-2}\right)\end{array}$ & $\begin{array}{c}\text { Broadleaves } \\
\text { dry weight } \\
\left(\mathrm{g} \mathrm{m}^{-2}\right)\end{array}$ & $\begin{array}{c}\text { Total weeds } \\
\text { dry weight ( } \mathrm{g} \\
\left.\mathrm{m}^{-2}\right)\end{array}$ \\
\hline 1- Pendimethalin $50 \% \mathrm{EC}$ & 2.023 & 4 & $\begin{array}{c}104.67 \\
(10.25 \mathrm{c}) \\
\end{array}$ & $\begin{array}{c}27.33 \\
(5.27 \mathrm{c}) \\
\end{array}$ & $\begin{array}{c}132.00 \\
(11.53 \mathrm{c}) \\
\end{array}$ & $\begin{array}{c}48.10 \\
(6.96 \mathrm{c}) \\
\end{array}$ & $\begin{array}{c}22.57 \\
(4.74 \mathrm{~cd}) \\
\end{array}$ & $\begin{array}{c}70.67 \\
(8.47 \mathrm{c}) \\
\end{array}$ \\
\hline 2- Thiobencarb $50 \%$ EC & 3.57 & 4 & $\begin{array}{c}304.00 \\
(17.45 \mathrm{~b})\end{array}$ & $\begin{array}{c}124.00 \\
(11.15 \mathrm{~b})\end{array}$ & $\begin{array}{c}428.00 \\
(20.70 \mathrm{~b})\end{array}$ & $\begin{array}{c}342.00 \\
(18.51 \mathrm{~b})\end{array}$ & $\begin{array}{c}192.00 \\
(13.87 \mathrm{~b})\end{array}$ & $\begin{array}{c}534.00 \\
(23.13 \mathrm{~b})\end{array}$ \\
\hline 3- Pendimethalin $f b$ penoxsulam & $2.023 f b 0.0238$ & 15 & $\begin{array}{c}60.33 \\
(7.80 \mathrm{e})\end{array}$ & $\begin{array}{c}9.33 \\
(3.11 \mathrm{fg})\end{array}$ & $\begin{array}{c}69.67 \\
(8.40 \mathrm{ef}) \\
\end{array}$ & $\begin{array}{c}28.07 \\
(5.33 \mathrm{~d})\end{array}$ & $\begin{array}{l}12.86 \\
(3.62 \mathrm{e})\end{array}$ & $\begin{array}{l}40.93 \\
(6.40 \mathrm{f})\end{array}$ \\
\hline 4- Pendimethalin $f b$ bispyribac-sodium & $2.023 f b 0.0381$ & 22 & $\begin{array}{l}58.40 \\
(7.67 \mathrm{e})\end{array}$ & $\begin{array}{c}8.73 \\
(3.02 \mathrm{fg})\end{array}$ & $\begin{array}{l}67.13 \\
(8.23 \mathrm{f})\end{array}$ & $\begin{array}{l}21.53 \\
(4.67 \mathrm{e})\end{array}$ & $\begin{array}{c}15.45 \\
(3.99 \mathrm{de})\end{array}$ & $\begin{array}{l}36.98 \\
(6.10 \mathrm{f})\end{array}$ \\
\hline 5- Pendimethalin $f b$ fenoxaprop-ethyl & $2.023 \mathrm{fb} 0.0625$ & 35 & $\begin{array}{l}58.00 \\
(7.64 \mathrm{e})\end{array}$ & $\begin{array}{c}16.33 \\
\text { (4.09 def) }\end{array}$ & $\begin{array}{l}74.33 \\
(8.70 \mathrm{e})\end{array}$ & $\begin{array}{l}20.80 \\
(4.60 \mathrm{e})\end{array}$ & $\begin{array}{l}19.43 \\
\text { (4.46 de }\end{array}$ & $\begin{array}{l}40.23 \\
(6.37 \mathrm{f})\end{array}$ \\
\hline 6- Thiobencarb $f b$ penoxsulam & $3.57 f b 0.0238$ & 15 & $\begin{array}{c}77.67 \\
(8.83 d)\end{array}$ & $\begin{array}{c}19.67 \\
\text { (4.49 cde) }\end{array}$ & $\begin{array}{c}97.33 \\
(9.87 \mathrm{~d})\end{array}$ & $\begin{array}{c}42.67 \\
(6.54 \mathrm{c}) \\
\end{array}$ & $\begin{array}{c}13.18 \\
(3.64 \mathrm{e})\end{array}$ & $\begin{array}{c}55.84 \\
(7.50 \mathrm{~d})\end{array}$ \\
\hline 7- Thiobencarb $f b$ bispyribac-sodium & $3.57 f b 0.0381$ & 22 & $\begin{array}{c}102.67 \\
(10.15 \mathrm{c})\end{array}$ & $\begin{array}{c}25.33 \\
(5.07 \mathrm{~cd})\end{array}$ & $\begin{array}{c}128.00 \\
(11.37 \mathrm{c})\end{array}$ & $\begin{array}{c}28.20 \\
(5.35 \mathrm{~d})\end{array}$ & $\begin{array}{c}17.89 \\
(4.27 \mathrm{de})\end{array}$ & $\begin{array}{c}46.08 \\
(6.83 \mathrm{e})\end{array}$ \\
\hline 8- Thiobencarb $f b$ fenoxaprop-ethyl & $3.57 f b 0.0625$ & 35 & $\begin{array}{c}78.00 \\
(8.83 \mathrm{~d})\end{array}$ & $\begin{array}{c}24.67 \\
(5.01 \mathrm{~cd})\end{array}$ & $\begin{array}{c}102.67 \\
(10.13 \mathrm{~d})\end{array}$ & $\begin{array}{c}25.40 \\
(5.09 \mathrm{de})\end{array}$ & $\begin{array}{c}29.51 \\
(5.47 \mathrm{c})\end{array}$ & $\begin{array}{c}54.91 \\
(7.43 \mathrm{~d})\end{array}$ \\
\hline $\begin{array}{l}\text { Pendimethalin } f b \text { penoxsulam + } \\
\text { halosulfuron }\end{array}$ & $\begin{array}{c}2.023 \mathrm{fb} \\
0.0238+0.0357\end{array}$ & 15 & $\begin{array}{c}4.67 \\
(2.27 \mathrm{~g})\end{array}$ & $\begin{array}{c}2.33 \\
(1.65 \mathrm{hi})\end{array}$ & $\begin{array}{c}7.00 \\
(2.80 \mathrm{i})\end{array}$ & $\begin{array}{l}4.27 \\
(2.17 \mathrm{f})\end{array}$ & $\begin{array}{c}1.13 \\
(1.23 \mathrm{~g})\end{array}$ & $\begin{array}{c}5.40 \\
(2.53 \mathrm{~g})\end{array}$ \\
\hline $\begin{array}{l}\text { Pendimethalin } f b \text { bispyribac-sodium }+ \\
\text { halosulfuron }\end{array}$ & $\begin{array}{c}2.023 \mathrm{fb} \\
0.0381+0.0357\end{array}$ & 22 & $\begin{array}{l}3.67 \\
(2.04 \mathrm{~g})\end{array}$ & $\begin{array}{c}1.33 \\
(1.27 \mathrm{i})\end{array}$ & $\begin{array}{c}5.00 \\
(2.40 \mathrm{i})\end{array}$ & $\begin{array}{c}3.73 \\
(2.05 \mathrm{f})\end{array}$ & $\begin{array}{c}2.20 \\
(1.63 \mathrm{fg})\end{array}$ & $\begin{array}{c}5.93 \\
(2.63 \mathrm{~g})\end{array}$ \\
\hline $\begin{array}{l}\text { Pendimethalin } f b \text { fenoxaprop-ethyl }+ \\
\text { alosulfuron }\end{array}$ & $\begin{array}{c}2.023 f b \\
0.0625+0.0357\end{array}$ & 35 & $\begin{array}{c}3.33 \\
(1.95 \mathrm{~g})\end{array}$ & $\begin{array}{c}2.00 \\
(1.56 \mathrm{hi})\end{array}$ & $\begin{array}{c}5.33 \\
(2.47 \mathrm{i})\end{array}$ & $\begin{array}{c}2.40 \\
(1.69 \mathrm{f})\end{array}$ & $\begin{array}{l}3.83 \\
(2.08 \mathrm{f})\end{array}$ & $\begin{array}{c}6.23 \\
(2.67 \mathrm{~g})\end{array}$ \\
\hline $\begin{array}{l}\text { Thiobencarb } f b \text { penoxsulam }+ \\
\text { halosulfuron }\end{array}$ & $\begin{array}{c}3.57 \mathrm{fb} \\
0.0238+0.0357\end{array}$ & 15 & $\begin{array}{l}21.87 \\
(4.73 \mathrm{f})\end{array}$ & $\begin{array}{c}5.63 \\
(2.46 \mathrm{gh})\end{array}$ & $\begin{array}{c}27.50 \\
(5.33 \mathrm{gh})\end{array}$ & $\begin{array}{c}4.27 \\
(2.17 \mathrm{f})\end{array}$ & $\begin{array}{c}1.87 \\
(1.53 \mathrm{fg})\end{array}$ & $\begin{array}{c}6.13 \\
(2.70 \mathrm{~g})\end{array}$ \\
\hline $\begin{array}{l}\text { Thiobencarb } f b \text { bispyribac-sodium + } \\
\text { halosulfuron }\end{array}$ & $\begin{array}{c}3.57 \mathrm{fb} \\
0.0381+0.0357\end{array}$ & 22 & $\begin{array}{l}20.60 \\
(4.59 \mathrm{f})\end{array}$ & $\begin{array}{c}6.07 \\
(2.55 \mathrm{gh})\end{array}$ & $\begin{array}{l}26.67 \\
(5.27 \mathrm{~h})\end{array}$ & $\begin{array}{c}4.27 \\
(2.17 \mathrm{f})\end{array}$ & $\begin{array}{c}2.60 \\
(1.74 \mathrm{fg})\end{array}$ & $\begin{array}{c}6.87 \\
(2.80 \mathrm{fg})\end{array}$ \\
\hline $\begin{array}{l}\text { Thiobencarb } f b \text { fenoxaprop-ethyl + } \\
\text { halosulfuron }\end{array}$ & $\begin{array}{c}3.57 \mathrm{fb} \\
0.0625+0.0357\end{array}$ & 35 & $\begin{array}{c}19.96 \\
(4.52 \mathrm{f})\end{array}$ & $\begin{array}{c}11.34 \\
(3.44 \mathrm{efg})\end{array}$ & $\begin{array}{c}31.33 \\
(5.70 \mathrm{~g}) \\
\end{array}$ & $\begin{array}{c}3.73 \\
(2.05 \mathrm{f}) \\
\end{array}$ & $\begin{array}{c}4.63 \\
(2.27 \mathrm{f}) \\
\end{array}$ & $\begin{array}{c}8.37 \\
(3.07 \mathrm{fg})\end{array}$ \\
\hline 15- Weedy check (untreated) & 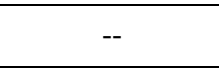 & -- & $\begin{array}{c}1273.33 \\
(35.68 \mathrm{a})\end{array}$ & $\begin{array}{c}523.99 \\
(22.85 \mathrm{a})\end{array}$ & $\begin{array}{l}1797.330 \\
(42.43 \mathrm{a})\end{array}$ & $\begin{array}{c}1168.00 \\
(34.18 \mathrm{a})\end{array}$ & $\begin{array}{c}808.67 \\
(28.43 \mathrm{a})\end{array}$ & $\begin{array}{c}1976.67 \\
(44.50 \mathrm{a})\end{array}$ \\
\hline $\operatorname{LSD}_{0.05}$ & - & - & 0.643 & 1.067 & 0.402 & 0.567 & 0.761 & 0.377 \\
\hline
\end{tabular}

Means of transformed data followed by the same letter are not significantly different at $5 \%$ level, using Duncan's Multiple Range Test. ai $=$ active ingredient. DAS= days after seeding. 


\section{B- Rice studied traits:}

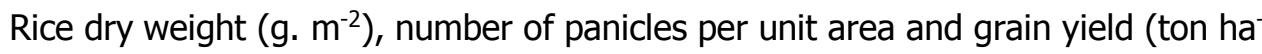
${ }^{1}$ ) were determined for rice to reflect the effect of tested factors on rice growth and yield. Results will be presented as follow:

\section{B.1. Effect of pre and post-emergence weed control treatments on dry} weight, number of panicles per unit area and rice grain yield during 2015 and 2016 seasons.

Table 3. Dry weight, number of panicles $\mathrm{m}^{-2}$ and grain yield of rice as affected by weed control treatments during 2015 and 2016 seasons.

\begin{tabular}{|c|c|c|c|c|c|c|}
\hline \multirow[b]{2}{*}{ Treatment } & \multicolumn{3}{|c|}{2015 season } & \multicolumn{3}{|c|}{2016 season } \\
\hline & $\begin{array}{l}\text { Rice dry } \\
\text { weight } \\
\left(\mathrm{g} \mathrm{m}^{-2}\right)\end{array}$ & $\begin{array}{c}\text { No. of } \\
\text { panicles } \\
\left(\mathrm{m}^{-2}\right)\end{array}$ & $\begin{array}{l}\text { Rice grain } \\
\text { yield } \\
\text { (ton ha }{ }^{-1} \text { ) }\end{array}$ & $\begin{array}{c}\text { Rice dry } \\
\text { weight } \\
\left(\mathrm{g} \mathrm{m}^{-2}\right)\end{array}$ & $\begin{array}{c}\text { No. of } \\
\text { panicles } \\
\left(\mathrm{m}^{-2}\right)\end{array}$ & $\begin{array}{c}\text { Rice grain } \\
\text { yield } \\
\text { ton ha-1 }^{-1}\end{array}$ \\
\hline 1- Pendimethalin $50 \%$ EC & $1020.00 \mathrm{~h}$ & $220.0 \mathrm{~g}$ & $5.003 \mathrm{e}$ & $1056.00 \mathrm{~d}$ & $272.0 \mathrm{~g}$ & $5.437 \mathrm{~g}$ \\
\hline 2- Thiobencarb 50\% EC & $640.0 \mathrm{i}$ & $144.0 \mathrm{~h}$ & $1.467 \mathrm{f}$ & $713.67 \mathrm{e}$ & $205.3 \mathrm{~h}$ & $2.303 \mathrm{~h}$ \\
\hline 3- Pendimethalin $f b$ penoxsulam & $1373.31 \mathrm{de}$ & 440.7 e & $6.869 \mathrm{~d}$ & $1369.33 \mathrm{bc}$ & $502.7 d$ & $8.340 \mathrm{~cd}$ \\
\hline $\begin{array}{l}\text { 4- Pendimethalin } f b \text { bispyribac- } \\
\text { sodium }\end{array}$ & 1300.0 ef & $476.6 \mathrm{~d}$ & $7.952 b c$ & $1346.00 \mathrm{bc}$ & $510.6 d$ & $8.538 \mathrm{c}$ \\
\hline $\begin{array}{l}\text { 5- Pendimethalin } f b \text { fenoxaprop- } \\
\text { ethyl }\end{array}$ & 1353.33 e & $492.0 \mathrm{~d}$ & $7.915 \mathrm{c}$ & $1306.67 b c$ & $517.3 \mathrm{~d}$ & $7.646 \mathrm{e}$ \\
\hline Thiobencarb $f b$ penoxsulam & $1213.33 \mathrm{fg}$ & $418.7 \mathrm{f}$ & $6.380 \mathrm{~d}$ & $1269.33 \mathrm{c}$ & $416.0 \mathrm{f}$ & $7.029 \mathrm{f}$ \\
\hline $\begin{array}{l}\text { 7- Thiobencarb } f b \text { bispyribac- } \\
\text { sodium }\end{array}$ & 1293.33 ef & $443.3 \mathrm{e}$ & $6.527 \mathrm{~d}$ & $1326.67 \mathrm{bc}$ & $420.7 \mathrm{f}$ & $6.835 f$ \\
\hline 8- Thiobencarb $f b$ fenoxaprop-ethyl & $1173.32 \mathrm{~g}$ & $437.3 \mathrm{e}$ & $6.417 \mathrm{~d}$ & $1265.33 c$ & 473.7 e & $6.744 f$ \\
\hline $\begin{array}{l}\text { 9- Pendimethalin } f b \text { penoxsulam + } \\
\text { halosulfuron }\end{array}$ & $1753.33 \mathrm{a}$ & $576.0 \mathrm{a}$ & $9.371 \mathrm{a}$ & $1656.67 a$ & $624.7 a$ & $9.255 a b$ \\
\hline $\begin{array}{l}\text { 10- Pendimethalin } f b \text { bispyribac- } \\
\text { sodium + halosulfuron }\end{array}$ & $1660.0 \mathrm{~b}$ & $594.7 \mathrm{a}$ & $9.296 a$ & $1541.33 \mathrm{a}$ & $642.0 \mathrm{a}$ & $9.449 a$ \\
\hline $\begin{array}{l}\text { 11- Pendimethalin } f b \text { fenoxaprop- } \\
\text { ethyl + halosulfuron }\end{array}$ & $1660.0 \mathrm{~b}$ & $586.7 a$ & $9.147 a$ & $1618.67 a$ & $636.67 a$ & $9.221 a b$ \\
\hline $\begin{array}{l}\text { 12- Thiobencarb } f b \text { penoxsulam }+ \\
\text { halosulfuron }\end{array}$ & $1453.31 \mathrm{~cd}$ & $528.0 \mathrm{bc}$ & $8.470 \mathrm{~b}$ & $1368.67 \mathrm{bc}$ & $561.3 \mathrm{C}$ & $8.287 \mathrm{~cd}$ \\
\hline $\begin{array}{l}\text { 13- Thiobencarb fb bispyribac- } \\
\text { sodium + halosulfuron }\end{array}$ & $1513.33 \mathrm{c}$ & $541.3 b$ & $8.470 \mathrm{~b}$ & $1361.33 b c$ & $560.0 \mathrm{C}$ & $8.125 d$ \\
\hline $\begin{array}{l}\text { 14- Thiobencarb fb fenoxaprop- } \\
\text { ethyl + halosulfuron }\end{array}$ & $1513.22 \mathrm{C}$ & $520.0 \mathrm{c}$ & $8.177 \mathrm{bc}$ & $1407.33 b$ & $589.3 b$ & $9.002 b$ \\
\hline 15- Weedy check (untreated) & $420.00 j$ & $85.3 \mathrm{i}$ & $1.063 \mathrm{f}$ & $377.33 \mathrm{f}$ & $114.7 i$ & $0.587 \mathrm{i}$ \\
\hline $\mathrm{LSD}_{0.05}$ & 90.07 & 18.65 & 0.481 & 113.7 & 25.26 & 0.308 \\
\hline
\end{tabular}

In a column, means followed by the same letter are not significantly different at $5 \%$ level, using Duncan's Multiple Range Test. 
It is clear from data in Table (3) that chemical weed control by both pre and post-emergence herbicides significantly increased rice dry weight as compared to untreated plots. The application of pendimethalin herbicide at 4 DAS recorded the highest dry weight of rice in the two seasons of study followed by thiobencarb $50 \%$ EC, while the lowest dry weight of rice plants was scored under un-treated plots (control) in 2015 and 2016 seasons. The highest dry weight of rice was obtained by spraying pendimethalin herbicide at 4 DAS followed by penoxsulam +halosulfuron treatment in 2015 and 2016 seasons without significant differences between pendimethalin $f b$ bispyribac-sodium +halosulfuron and pendimethalin $f b$ fenoxaprop +halosulfuron in 2016 season. On the other side, weedy check plots recorded the lowest dry weight of rice $\left(\mathrm{g} \mathrm{m}^{-2}\right)$ over the two seasons. These results are in conformity with the findings obtained by Walia et al. (2008) and Anwar et al. (2013).

In respect to number of panicles per unit area and rice grain yield, it is evident from data in Table (3) that number of panicles per unit area and rice grain was significantly affected by weed control treatments over the two seasons of study. Using pendimethalin as a pre-emergence herbicide followed by any of penoxsulam $2.5 \%$ or bispyribac-sodium $2 \%$ or fenoxaprop-ethyl $7.5 \%$ mixed with halosulfuron $75 \%$ as post-emergence weed control treatments scored the highest values of number of panicles $\mathrm{m}^{-2}$ and grain yield of rice during 2015 and 2016 seasons.

On the opposite, the lowest values of number of panicles $\mathrm{m}^{-2}$ and grain yield were recorded under un-treated plots in both seasons of study. The high yield may be due to high efficiency of pendimethalin as pre-emergence herbicide in suppress weed seed germination then reducing weed competition resulting in better growth conditions for rice such as water, macro and micro elements uptake and light which reflex on the high grain yield of rice. Similar results were obtained by Mahajan and Chauhan, 2015. Singh et al., 2006 and Chauhan et al., 2013 reported that the mixture application of herbicides significantly reduced weed biomass and save the best rice growth demands to gain the highest yield of rice than the single application of herbicides.

\section{B.2. Effect of pre and post-emergence weed control treatments on dry weight, number of panicles per unit area and rice grain yield during 2015 and 2016 seasons.}

Data on panicle weight, number of filled grain / panicle and 1000-grain weight of rice as affected by pre and post-emergence weed control treatments during 2015 and 2016 seasons are presented in Figures 1, 2 and 3.

All herbicides (pre and post-emergence) treatments significantly increased panicle weight, 1000-grain weight and number of filled grain / panicle of rice as 
compared to weedy check (untreated plots) during the two seasons of study. The highest values of these characters were recorded with pre-emergence herbicide application of pendimethalin at $2.023 \mathrm{~kg}^{\text {ai }} \mathrm{ha}^{-1}$ followed by post-emergence application of penoxsulam or bispyribac-sodium or fenoxaprop at the recommended dose in mixture with halosulfuron at the recommended dose, while thiobencarb application at 4 DAS followed by applying the same abovementioned post-emergence weed control treatments ranked second. On the other hand, weedy check plots recorded the lowest values of panicle weight, 1000-grain weight and number of filled grain per panicle of rice during 2015 and 2016 seasons. The increasing in these characters due to reduced completion as a results of effective weed control by herbicides. Similar results were obtained by Walia et al. (2012).

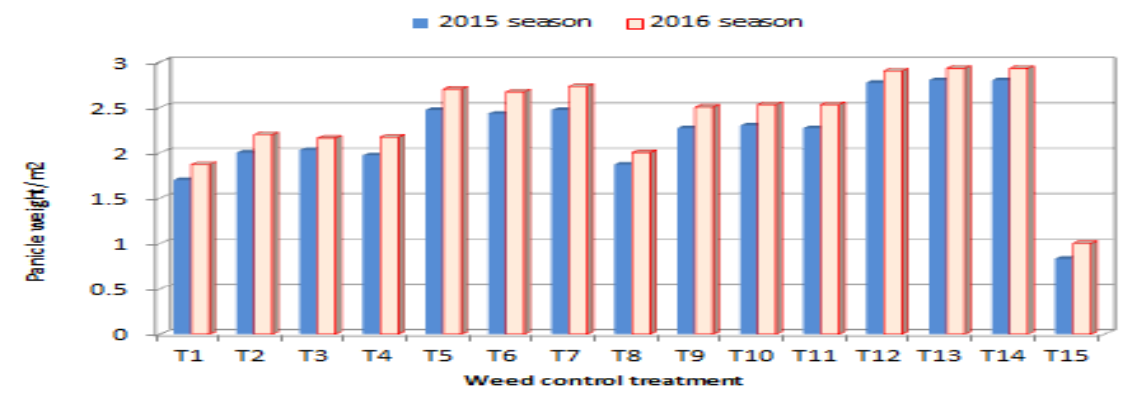

Fig. 1. Panicle weight of rice as affected by weed control treatments in 2015 and 2016 seasons

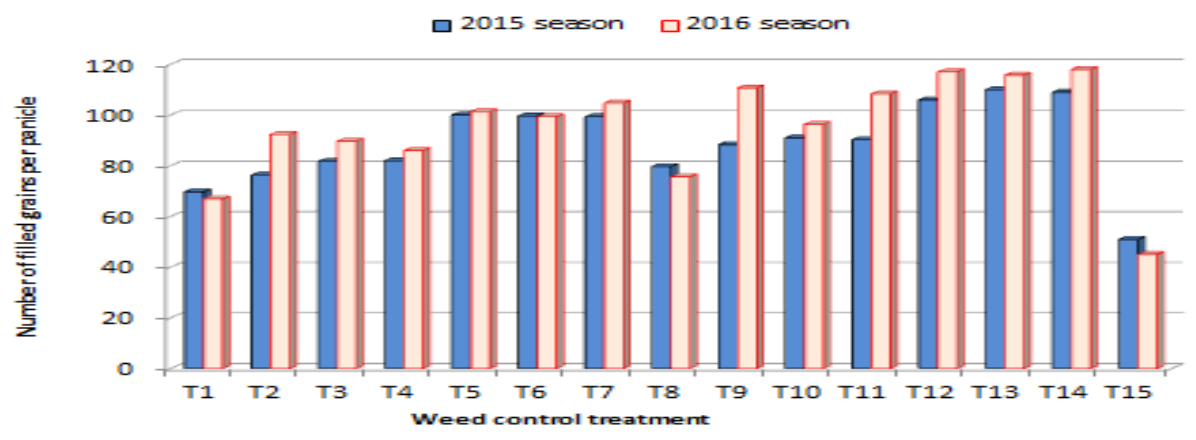

Fig. 2. Number of filled grains per panicle of rice as affected by weed control in treatments in 2015 and 2016 seasons 


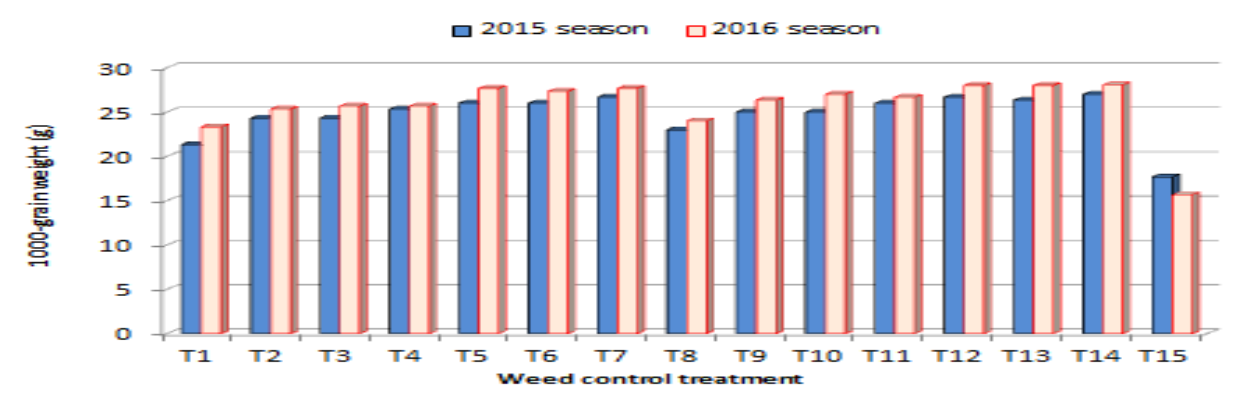

Fig. 3. 1000-grain weight (g) of rice as affected by weed control in treatments in 2015 and 2016 seasons

$\mathbf{T}_{\mathbf{1}}=$ thiobencarb, $\mathbf{T}_{\mathbf{2}}=$ thiobencarb $\boldsymbol{f} \boldsymbol{b}$ penoxsulam, $\mathbf{T}_{\mathbf{3}}=$ thiobencarb $\boldsymbol{f} \boldsymbol{b}$ bispyribac, $\mathbf{T}_{\mathbf{4}}=$ thiobencarb $\boldsymbol{f} \boldsymbol{b}$ fenoxaprop, $\mathbf{T}_{\mathbf{5}}=$ thiobencarb $\boldsymbol{f} \boldsymbol{b}$ penoxsulam + halosulfuron, $\mathbf{T}_{\mathbf{6}}=$ thiobencarb $\boldsymbol{f} \boldsymbol{b}$ bispyribac + halosulfuron, $\mathbf{T}_{\mathbf{7}}=$ thiobencarb $\boldsymbol{f b}$ fenoxaprop + halosulfuron, $\mathbf{T}_{\mathbf{8}}=$ pendimethalin, $\mathbf{T}_{\mathbf{9}}=$ pendimethalin $\boldsymbol{f b}$ penoxsulam, $\mathbf{T}_{\mathbf{1 0}}=$ pendimethalin $\boldsymbol{f} \boldsymbol{b}$ bispyribac, $\mathbf{T}_{\mathbf{1 1}}=$ pendimethalin $\boldsymbol{f} \boldsymbol{b}$ fenoxaprop, $\mathbf{T}_{\mathbf{1 2}}=$ pendimethalin $\boldsymbol{f} \boldsymbol{b}$ penoxsulam + halosulfuron, $\mathbf{T}_{\mathbf{1 3}}=$ pendimethalin $\boldsymbol{f} \boldsymbol{b}$ bispyribac + halosulfuron, $\mathbf{T}_{\mathbf{1 4}}=$ pendimethalin $\boldsymbol{f} \boldsymbol{b}$ fenoxaprop + halosulfuron, and $\mathbf{T}_{\mathbf{1 5}}=$ Weedy check (control).

C- Correlation coefficients among studied traits of weeds and rice) in 2015 and 2016 seasons.

Data in Table (4) are showed the correlation among studied traits of weeds and rice growth, yield and some yield attributes in the two seasons of study. 
Table 4. Correlation coefficients among studied traits of weeds and rice in 2015 and

\begin{tabular}{|c|c|c|c|c|c|c|c|c|}
\hline & $\begin{array}{c}\text { Total } \\
\text { weeds } \\
\text { dry } \\
\text { weight }\end{array}$ & $\begin{array}{c}\text { Total } \\
\text { weeds } \\
\text { fresh } \\
\text { weight }\end{array}$ & $\begin{array}{l}\text { Rice dry } \\
\text { weight }\end{array}$ & $\begin{array}{c}\text { Number } \\
\text { of } \\
\text { panicle/m² }\end{array}$ & $\begin{array}{l}\text { Panicle } \\
\text { weight }\end{array}$ & $\begin{array}{l}\text { Filled } \\
\text { grains / } \\
\text { panicle }\end{array}$ & $\begin{array}{l}\text { 1000- } \\
\text { grain } \\
\text { weight }\end{array}$ & $\begin{array}{l}\text { Grain } \\
\text { yield }\end{array}$ \\
\hline & \multicolumn{8}{|c|}{2015 season } \\
\hline $\begin{array}{l}\text { Total weeds } \\
\text { dry weight }\end{array}$ & 1 & $0.962 * *$ & $\begin{array}{c}- \\
0.788^{* *}\end{array}$ & $-0.749 * *$ & $\overline{-}-\overline{3} 4 * *$ & $\begin{array}{c}- \\
0.768^{* *}\end{array}$ & $\begin{array}{c}- \\
0.946 * *\end{array}$ & $\begin{array}{c}- \\
0.771^{* *}\end{array}$ \\
\hline $\begin{array}{l}\text { Total weeds } \\
\text { fresh weight }\end{array}$ & $0.962 * *$ & 1 & $0 . \overline{10 * *}$ & $-0.879 * *$ & $\begin{array}{c}- \\
0.933^{*} * \\
\end{array}$ & $\begin{array}{c}-\overline{-} \\
0.898^{* *}\end{array}$ & $\begin{array}{c}-\overline{-} \\
0.982^{* *}\end{array}$ & $\begin{array}{c}- \\
0.897 * *\end{array}$ \\
\hline $\begin{array}{l}\text { Rice dry } \\
\text { weight }\end{array}$ & $\begin{array}{c}- \\
0.788^{*} *\end{array}$ & $0 . \overline{10 * *}$ & 1 & $0.956 * *$ & $0.926 * *$ & $0.947 * *$ & $0.898 * *$ & $0.968 * *$ \\
\hline $\begin{array}{l}\text { Number of } \\
\text { panicle/m² }\end{array}$ & $\begin{array}{c}-\overline{-} \\
0.749 * *\end{array}$ & $\begin{array}{c}- \\
0.879 * *\end{array}$ & $0.956 * *$ & 1 & $0.913 * *$ & $0.914 * *$ & $0.880 * *$ & $0.973 * *$ \\
\hline $\begin{array}{l}\text { Panicle } \\
\text { weight }\end{array}$ & $0.834 * *$ & $0.933 * *$ & $0.926 * *$ & $0.913 * *$ & 1 & $0.956 * *$ & $0.928^{* *}$ & $0.916 * *$ \\
\hline $\begin{array}{l}\text { Filled grains } \\
\text { per panicle }\end{array}$ & $\begin{array}{c}- \\
0.768^{* *} \\
\end{array}$ & $\begin{array}{c}- \\
0.898^{* *} \\
\end{array}$ & $0.947 * *$ & $0.914^{* *}$ & $0.956 * *$ & 1 & $0.896 * *$ & $0.925 * *$ \\
\hline $\begin{array}{c}\text { 1000-grain } \\
\text { weight }\end{array}$ & $0.946^{*} * *$ & $0.982 * *$ & $0.898^{* *}$ & $0.880 * *$ & $0.928 * *$ & $0.896 * *$ & 1 & $0.882 * *$ \\
\hline \multirow[t]{2}{*}{ Grain yield } & $\begin{array}{c}- \\
0.771 * *\end{array}$ & $\begin{array}{c}- \\
0.897 * *\end{array}$ & $0.968 * *$ & $0.973 * *$ & $0.916^{* *}$ & $0.925^{* *}$ & $0.882 * *$ & 1 \\
\hline & \multicolumn{8}{|c|}{2016 season } \\
\hline $\begin{array}{l}\text { Total weeds } \\
\text { dry weight }\end{array}$ & 1 & $0.987 * *$ & $\begin{array}{c}- \\
0.847 * *\end{array}$ & $-0.743 * *$ & $\begin{array}{c}- \\
0.813 * *\end{array}$ & $\begin{array}{c}- \\
0.755^{*} * \\
\end{array}$ & $\begin{array}{c}- \\
0.846 * *\end{array}$ & $\begin{array}{c}- \\
0.837 * * \\
\end{array}$ \\
\hline $\begin{array}{l}\text { Total weeds } \\
\text { fresh weight }\end{array}$ & $0.987 * *$ & 1 & -0.906 & $-0.826 * *$ & $\begin{array}{c}- \\
0.867 * *\end{array}$ & $\begin{array}{c}-\overline{-} \\
0.813^{*} * \\
\end{array}$ & $\begin{array}{c}- \\
0.893 * * \\
\end{array}$ & -0.905 \\
\hline $\begin{array}{c}\text { Rice dry } \\
\text { weight }\end{array}$ & $\begin{array}{c}- \\
0.847 * *\end{array}$ & $\begin{array}{c}- \\
0.906 * *\end{array}$ & 1 & $0.930 * *$ & $0.915^{* *}$ & $0.913^{* *}$ & $0.907 * *$ & $0.963 * *$ \\
\hline $\begin{array}{l}\text { Number of } \\
\text { panicle/m² }\end{array}$ & $\begin{array}{c}- \\
0.743 * * \\
\end{array}$ & $\begin{array}{c}- \\
0.826 * * \\
\end{array}$ & $0.930 * *$ & 1 & $0.944 * *$ & $0.917 * *$ & $0.905^{* *}$ & $0.961 * *$ \\
\hline $\begin{array}{l}\text { Panicle } \\
\text { weight }\end{array}$ & $\begin{array}{c}- \\
0.813^{*} *\end{array}$ & $\begin{array}{c}- \\
0.867 * *\end{array}$ & $0.915^{* *}$ & $0.944 * *$ & 1 & $0.922 * *$ & $0.892 * *$ & $0.933 * *$ \\
\hline $\begin{array}{l}\text { Filled grains } \\
\text { per panicle }\end{array}$ & $0.755^{*} * *$ & $0.813 * *$ & $0.913^{* *}$ & $0.917 * *$ & $0.922 * *$ & 1 & $0.867 * *$ & $0.916^{* *}$ \\
\hline $\begin{array}{c}\text { 1000-grain } \\
\text { weight }\end{array}$ & $\begin{array}{c}- \\
0.846 * * \\
\end{array}$ & $0 . \overline{-}$ & $0.907 * *$ & $0.905^{* *}$ & $0.892^{* *}$ & $0.867^{* *} *$ & 1 & $0.911^{* *}$ \\
\hline Grain yield & $\begin{array}{c}- \\
0.837 * *\end{array}$ & $0 . \overline{-} * 5^{* *}$ & $0.963 * *$ & $0.961^{* *}$ & $0.933 * *$ & $0.916 * *$ & $0.911^{* *}$ & 1 \\
\hline
\end{tabular}

** indicated highly significant at 0.05 and 0.01 level of probability.

It is obvious from the obtained data that fresh and dry weights of total weeds were highly significantly positively correlated in 2015 and 2016 seasons. On the other hand, there were highly significantly negative correlations between both traits and rice dry weight, yield and yield attributes in both seasons of study. All rice studied traits showed highly significantly positively correlation between each other, while appeared highly significant negative correlations with total weeds fresh and dry weights in 2015 and 2016 seasons.

Grain yield of rice was highly significantly negatively correlated with both fresh and dry weights of total weeds in the two seasons of study. But highly significantly positively correlated with rice dry weight and panicles number per square meter $\left(r=0.968^{* *}, 0.963^{* *}\right.$ and $0.973 * *$ and $\left.0.961^{* *}\right)$ in 2015 and 2016 seasons, respectively. In addition the positive correlation with other yield components in the two seasons of study. Previous studies also revealed a negative correlation between rice yield and weed biomass in DSR (Chauhan et al., 2011). Rice grain yield was 
negatively and linearly associated with weed density and weed biomass. (Abdul Khaliq and Chauhan, 2014).

\section{CONCLUSION}

Based on the obtained results under study, it can be concluded that weed management in drill-seeded rice must be a sequential application of herbicides. Moreover, the application of herbicide mixtures as a post-emergence was more effective than single application. The maximum rice grain yield and best weed control were achieved by the application of pendimethalin $50 \% \mathrm{EC}$ at rate of $2.023 \mathrm{Kg}$ ai ha-1 at 4 DAS as pre-emergence herbicide followed by adding any of penoxsulam $2.5 \%$ OD or bispyribac-sodium $2 \%$ SL or fenoxaprop-ethyl $7.5 \%$ EW mixed with halosulfuron $75 \%$ WG at recommended doses as post-emergence herbicides at 15, 22 and 35 DAS, respectively.

\section{REFERENCES}

1. Abdul Khaliq, A. M. and B. S. Chauhan. 2014. Weed management in dry-seeded fine rice under varying row spacing in the rice-wheat system of Punjab, Pakistan. Plant Production Science, 17(4): 321-332.

2. Anwar, M. P.; A. S. Jauraimi; M. T. M. Mohamed; M. K. Uddin; B. Sanedan; A. Putech and A. Man. 2013. Integration of agronomic practices with herbicides for sustainable weed management in aerobic rice. Sci. World J., http://dx.doi.org/10.1155/2013/916408.

3. Awan, T. H.; P. C. Sta Cruz and B. S. Chauhan. 2015. Agronomic indices, growth, yield contributing traits, and yield of dry-seeded rice under varying herbicides. Field Crops Res., 177: 15-25.

4. Chauhan, B. S. 2012. Weed ecology and weed management strategies for dryseeded rice in Asia. Weed Technol. 26:1-13.

5. Chauhan, B. S.; A. S. K. Abeysekara; S. D. Kulatunga and U. B. Wickramu. 2013. Performance of different herbicides in dry-seeded rice in Sri-Lanka. Weed Technol., 27: 452-462.

6. Chauhan, B. S.; S. Ahmed and T. H. Awan. 2015. Performance of sequential herbicides in dry-seeded rice in Philippines. Crop Protection, 74: 124-130.

7. Chauhan, B.S.; V. P. Singh; A. Kumar and D. E. Johnson. 2011. Relations of rice seeding rates to crop and weed growth in aerobic rice. Field Crops Res. 121: 105115.

8. Duncan, D. B. 1955. Multiple range and multiple F-tests. Biometrics 11: 1-42.

9. El-Refaee, I. S.; R. A. Ebaid and I. M. El-Rewiny. 2006. Performance of rice (Oryza sativa L.) plant under different water regimes and methods of planting. Alex. J. Agric. Res., 51 (2): 47-55. 
10. FAOSTAT. 2011. FAO Statistical databases. Food and Agriculture Organization (FAO) of the United Nations, Rome. www.fao.org.

11. FAOSTAT. 2014. FAO Statistical database. Food and Agriculture Organization (FAO) of the United Nations, Rome. www.fao.org.

12. Ganie, Z. A.; S. Singh and S. Singh. 2014. Integrated weed management in dryseeded rice. Ind. J. Weed Sci., 46(2): 72-73.

13. Hongyan, L.; H. Saddam; M. Zheng; L. Sun; F. Shah; J. Huang; K. Cui and L. Nie. 2014. Progress and constraints of dry-seeded rice in China. J. of Food, Agric. \& Environ., 12(2): 465-472.

14. Mahajan, G. and B. S. Chauhan. 2013. Herbicide options for weed control in dryseeded aromatic rice in India. Weed Technol., 27: 682-689.

15. Mahajan, G. and B. S. Chauhan. 2015. Weed control in dry direct-seeded rice using tank mixtures of herbicides in South Asia. Crop Protection, 72: 90-96.

16. Mahajan, G. and B. S. Chauhan and D. E. Johnson. 2009. Weed management in aerobic rice in north western Indo-Gangetic Plains. J. Crop Improve. 23: 366-382.

17. Ranjit, J. D. 1997. Weeds and weed management in the rice-wheat system. Proceedings of the Rice-Wheat Research End-of-Project Workshop; Kathmandu (Nepal); 1-3 Oct.

18. Singh, A.; D. P. Nandal and S. S. Puina. 2017. Bio efficacy of sequential application of herbicides on weed and yield in direct seeded rice (Oryza sativa L.). Int. J. Curr. Microbial. App. Sci., 6(4): 900-905.

19. Singh, S.; L. Bhushan; j. K. Ladha; R. K. Gupta; A. N. Rao and B. Sivaprasad. 2006. Weed management in dry-seeded rice (Oryza sativa L.) cultivated in the furrow-irrigated raised-bed planting system. Crop Protection, 25: 487-495.

20. Singh, V.; M. L. Jat; Z. A. Ganie; B. S. Chauhan and R. K. Gupta. 2016. Herbicide option for effective weed management in dry direct-seeded rice under scented rice-wheat rotation of western Indo-Gang etic plains. Crop Protection, 81: 168176.

21. Snedecor, G. W. and W. G. Cochran. 1971. Statistical Methods. $\sigma^{\text {th }}$ ed., Iowa State Univ. Press Ames, USA.

22. Steel, R. G. and J. H. Torrie. 1960. Principles and procedures of statistics. McGraw Hill Book Company, INC. New York.

23. Walia, U. S.; M. S. Bhullar; N. Shelly; S. S. Walia. 2008. Control of complex weed flora of dry-seeded rice (Oryza sativa L.) with pre and post-emergence herbicides. Ind. J. Weed Sci., 40: 161-164.

24. Walia, U. S.; S. S. Walia; A. S. Sidhu and S. Nayyar. 2012. Bio efficacy of preand post-emergence herbicides in direct-seeded rice in central Punjab. Indian J., of Weed Sci., 44(1): 30-33. 


\section{التكامل بين مبيدات قبل وبعد الإبثاق في مكافحة حشائش الأرز التسطير}

\section{صبري صبحي محمد عبدالنبي، إبراهيم حمدي أبو الارج وأحمد مصطفى أحمد الغندور}

قسم بحوث الأرز - معهل بحوث المحاصيل الحقلية- مركز البحوث الزراعية- الجيزة - جمهورية مصر العربية

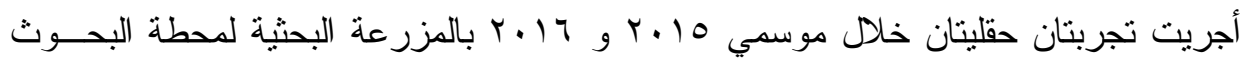
الزر اعية بسخا-كفر الثيخ-جمهورية مصر العربية للوصول لأفضل معاملات قبل وبعد الانبثاق مسن

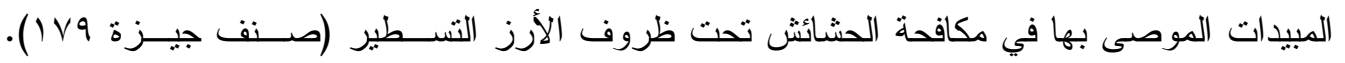
وكانت معاملات مكافحة الحشائش المدروسة كالاتي: مبيد ثيوبينكارب .0\% بمعدل r,OV كجم مادة

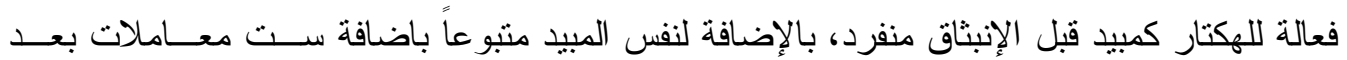

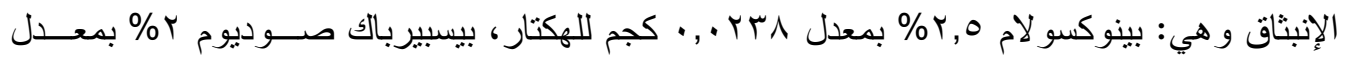

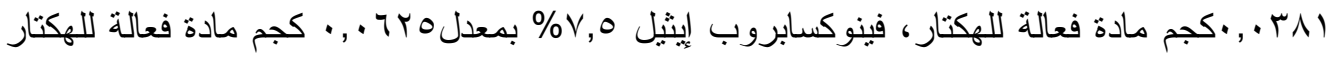

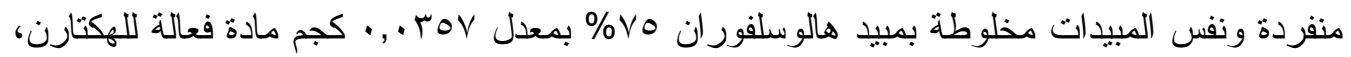

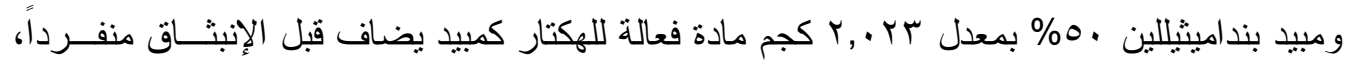
ومنبو عاً بنفس الست معاملات السابقة بعد الإنبثاق مقارنة بالقطع غير المعاملة بالمبيد (الكنتــرول).

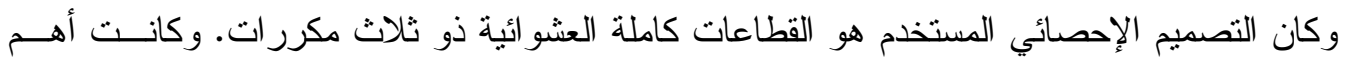

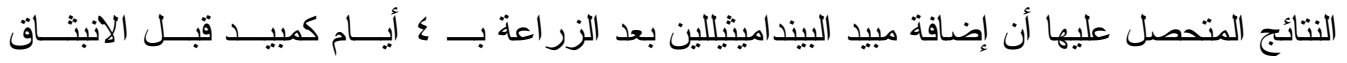

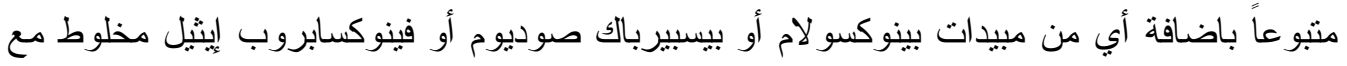

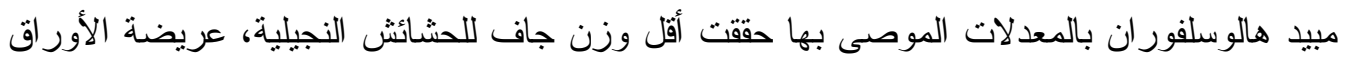
و الحشائش الكلية وكذللك أعلى مادة جافة وصفات المحصول ومكوناته للأرز خلال موسمي الزر اعة.

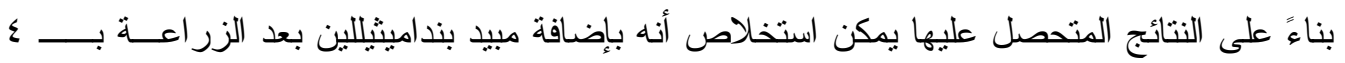

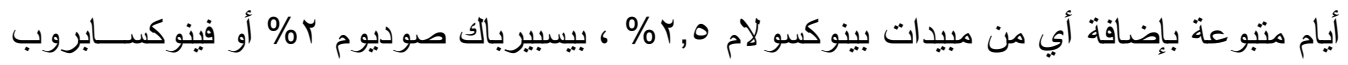

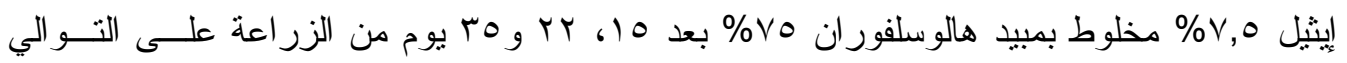

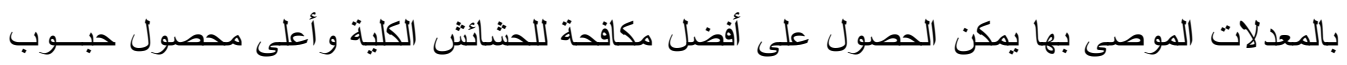
للأرز التسطير . بالات 